ISSN 1112-9867

http://www.jfas.info

\title{
WATER AND PHYSIOLOGICAL RESPONSES OF OKRA (Abelmoschus esculentus (L.) Moench) UNDER SALINE STRESS GROWN ON A BENTONIZED SUBSTRATE
}

\author{
H. Rahim Guealia ${ }^{1}$, M. Belkhodja ${ }^{2}$, H. A. Reguieg yssaad ${ }^{1}$, F. Z. Babou ${ }^{2}$ \\ ${ }^{1}$ Department of Biology, Laboratory of Biodiversity and Conservation of Water and Soils, \\ University of A.Benbadis/Mostaganem, Algeria \\ ${ }^{2}$ Department of Biology, Laboratory of Plant Physiology, University Oran 1 Ahmed Ben \\ Bella, Oran, Algeria
}

Received: 18 February 2017 / Accepted: 20 Jully 2017 / Published online: 01 September 2017

\begin{abstract}
In many arid and semi-arid regions of the world, Salinity has become an important problem for agricultural production. The objective of this study was to evaluate the effect of different treatments of $\mathrm{NaCl}$ (Control, $100 \mathrm{mM}$ and $300 \mathrm{mM}$ ) for 7 days, on young okra plants (Abelmoschus esculentus), grown in two types of substrate with bentonite (B) 7\% and without bentonite (WB) under controlled greenhouse conditions. The results showed that the two factors (salinity and bentonite) imposed in our study have a significant effect on the water status estimated by RWC, RWL and DHS in addition of the accumulation of the osmoregulator (proline, soluble sugars).
\end{abstract}

Keywords: okra, bentonite, salinity, water parameters, biochemical parameters.

Author Correspondence, e-mail: htcmangrove@gmail.com

doi: http://dx.doi.org/10.4314/jfas.v9i3.10

\section{INTRODUCTION}

Les modifications environnementales dues aux changements climatiques marqués depuis quelques décennies, se traduisent essentiellement par un déficit hydrique important et la 
progression de la salinité, notamment dans les régions arides et semi-arides [1,2]. Dans ces écosystèmes caractérisés par des périodes de sécheresses rigoureuses et fréquentes, qui constituent environ $2 / 3$ de la surface du globe terrestre [3]. La salinité prend de plus en plus d'ampleur. A l'échelle mondiale, il est estimé que presque $6 \%$ de terres sont affectées par le sel [4], dont 3.8\% sont situés en Afrique [5].

La salinité est l'un des facteurs écologiques susceptibles de limiter la croissance et le développement des plantes [6], en modifiant manifestement leur physiologie [7]. Elle entraine aussi un déficit hydrique chez les plantes sous forme de sécheresse physiologique [8]. Des fortes concentrations de $\mathrm{NaCl}$ dans la zone racinaire peuvent diminuer le potentiel hydrique du sol et la disponibilité de l'eau [9]. Cette insuffisance d'eau accessible par la plante provoque la déshydratation au niveau cellulaire, marqué par une diminution de contenu hydrique des feuilles, transpiration et l'assimilation hydrique par le système racinaire [10-12]. En effet, dans des conditions de stress salin les plantes développent des mécanismes de défense très complexes et varient d'une espèce à l'autre, voir d'une variété à l'autre dans la même espèce [13]. Afin d'atténuer l'effet du stress salin, les plantes sont capable de synthétiser des composés organiques dits osmoprotecteurs (ou solités compatibles), tels que les sucres solubles [14], les acides aminées et plus particulièrement la proline [15]. L'ajustement osmotique constitue un processus primordial qui permet à la cellule de maintenir sa turgescence [16]. D’autres mécanismes se manifestent lors du stress salin afin de limiter les pertes en eau, régulation stomatique [17], régulation phytohormonal [18], et le transport de l'eau [19].

Depuis de nombreuses années, l'Algérie fait partie du groupe de pays caractérisés par des sols salés [20]. Pour faire face à cette situation inquiétante, des actions à entreprendre en vue d'améliorer et réhabiliter ces écosystèmes, en utilisant des ressources naturelles disponibles, l'ajout de bentonite riche en argile dans le sol, ce qui permettrait d'augmenter à la fois son réserve en eau et sa capacité à retenir les éléments minéraux. Cette technique très ancienne est utilisée de façon traditionnelle dans le Sahara algérien ainsi qu'en Égypte [21].

Dans le présent travail on s'est intéressé à l'effet du stress salin sur quelques paramètres hydriques et biochimiques, d'une espèce appartient à la famille des malvacées, le gombo 
(Abelmoschus esculentus L.) est l'une des cultures horticoles les plus importantes dans de nombreux pays [22]. Conduit sur substrat bentonisé et soumis à différentes doses de $\mathrm{NaCl}$.

\section{MATERIEL ET MÉTHODES}

\subsection{Préparation de substrat de culture}

Le substrat de culture est constitué de sable tamisé et traité à l'esprit de sel afin d'éliminer les déchets organiques, puis lavé 6 fois à l'eau de robinet et enfin rincé à l'eau déminéralisée. La bentonite est additionnée au sable dans la proportion de 7\%. Les pots en plastique $(30 \mathrm{~cm}$ de profondeur et une capacité de $1.8 \mathrm{~kg}$ ) sont entreposés selon un dispositif au hasard, soit deux blocs de plantes répartis respectivement (le premier protocole au $\mathrm{NaCl}$ à 0,100 et $300 \mathrm{mM}$ en absence de bentonite et le second protocole en présence de bentonite à $7 \%$ dans les mêmes conditions de salinité). Trois répétions de plantes sont opérées pour chaque traitement.

Table 1. Composition chimique de la bentonite brute de Mzila/Mostaganem (ENOF, 1988)

\begin{tabular}{lccccccc}
\hline $\begin{array}{l}\text { Composants de la } \\
\text { bentonite }\end{array}$ & $\mathrm{SiO}_{2}$ & $\mathrm{Al}_{2} \mathrm{O}_{3}$ & $\mathrm{CaO}$ & $\mathrm{MgO}$ & $\mathrm{Na}_{2} \mathrm{O}$ & $\mathrm{K}_{2} \mathrm{O}$ & $\mathrm{FeO} 3$ \\
\hline Teneur en \% & 61.2 & 13.50 & 4.52 & 2.78 & 1.57 & 1.73 & 3.55
\end{tabular}

\subsection{Matériel végétal}

Les graines d'Abelmoschus esculentus L. récoltées de la région de Nechmaya (Wilaya de Guelma, Nord-Est Algérien), sont désinfectées par un lavage avec de l'hypochlorite de sodium (2\%) pendant quelques minutes, puis rincées abondamment à l'eau distillée pour éliminer l'eau de javel. Elles sont semées en alvéoles remplies de terreau jusqu'au stade plantule dans une serre contrôlée du laboratoire de Physiologie Végétale. Ensuite, les plantules sont repiquées dans les pots. Un arrosage tous les trois jours est opéré à la solution nutritive de Hoagland (1938) à 30\% de la capacité de rétention du substrat. Au 53ème jour, les plantes ont subi pendant une semaine un stress salin au $\mathrm{NaCl}$ à 100 et $300 \mathrm{mM}$. Les plantes témoins reçoivent de la solution nutritive de Hoagland à $60 \%$ de la capacité de rétention du substrat.

\subsection{LES MESURES EFFECTUEES}




\subsubsection{La teneur relative en eau (RWC)}

La teneur relative en eau a été mesurée sur la dernière feuille entièrement développée pour déterminer l'état hydrique des plantes, elle est calculée par la formule [23]:

$$
R W C(\%)=\frac{P f-P s}{P s a t-P s} \times 100
$$

Pf: poids frais; PS: poids sec; P sat: poids à saturation

\subsubsection{Le taux de déperdition de l'eau (RWL)}

La perte graduelle en eau est déterminée selon la formule de [23]:

$$
R W L=\frac{P i-P t}{S f \times T}
$$

Pi: poids initial obtenu après $48 \mathrm{~h}$ dans l'eau distillée à $4^{\circ} \mathrm{C}$ et à $1^{\prime}$ obscurité;

Sf: surface foliaire.

$\mathrm{Pt}$ : poids mesuré après transpiration durant 30 et $60 \mathrm{mn}$;

T: temps de transpiration.

La perte graduelle en eau (RWL) est exprimée en $\mathrm{mg}$ d'eau perdue $\mathrm{cm}^{-2}$. $\mathrm{Min}^{-1}$.

\subsubsection{Le déficit de saturation hydrique (DHS)}

Le DHS témoigne du niveau du stress hydrique auquel sont soumises les plantes, est évalué par l'écart entre la teneur en eau maximale obtenue à la pleine turgescence et la teneur en eau réelle, le tout exprimé en pourcentage (\%) de la teneur en eau maximale [24]. Le déficit de saturation hydrique (DSH) est obtenu par la formule suivante:

$$
\operatorname{DSH}(\%)=\frac{P T-P F}{P T-P S} \times 100
$$

PF: le poids de la feuille à l'état frais; PT: le poids turgescent; PS: le poids sec.

\subsubsection{Détermination de la proline libre}

L'extraction et le dosage de la proline des feuilles et des racines sont réalisés selon la méthode de [25], Le principe est la quantification de la réaction proline-ninhydrine par mesure spectrophotométrique à la longueur d'onde de 515nm. La proline se couple avec la ninhydrine en formant un complexe coloré. L'intensité de la coloration est proportionnelle à la quantité de proline. Les teneurs en proline sont exprmés en mg. $\mathrm{g}^{-1} \mathrm{PS}$.

\subsubsection{Détermination des sucres solubles}


L'extraction et le dosage sont fait par la méthode de [26] modifiée par [27], qui consiste à ajouter $4 \mathrm{ml}$ de réactif d'anthrone à $2 \mathrm{ml}$ de l'extrait éthanolique des échantillons frais. La DO

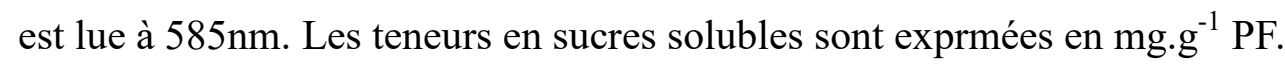

Une étude statistique comportant une analyse de la variance et le calcul de corrélations, est effectué par le logiciel statistica.

\section{RÉSULTATS}

\subsection{Teneur relative en eau ( $R W C$ )}

L'analyse statistique des résultats révèle un effet significatif de la salinité sur le contenu hydrique des feuilles de gombo $(p<0,005)$. Le RWC est influencé de façon hautement significative par l'interaction des deux facteurs étudiés $(p<0,005)$ (Tableau 2). Les résultats présentés dans la Figure1 mettent en évidence une réponse différente des plantes cultivées au sein des deux substrats. En effet, chez les plantes conduites sous substrat sans bentonite, la RWC des feuilles a diminué significativement de 4 et 13\% sous l'effet de 100 et $300 \mathrm{mM}$ $\mathrm{NaCl}$ respectivement. D'ailleurs, sous ces mêmes concentrations et en présence de bentonite à 7\%, le RWC des feuilles n'a subi aucune différence significative par rapport au témoin avec des taux de $88,3 \%$ pour les témoins contre 86,2 et $84,9 \%$.

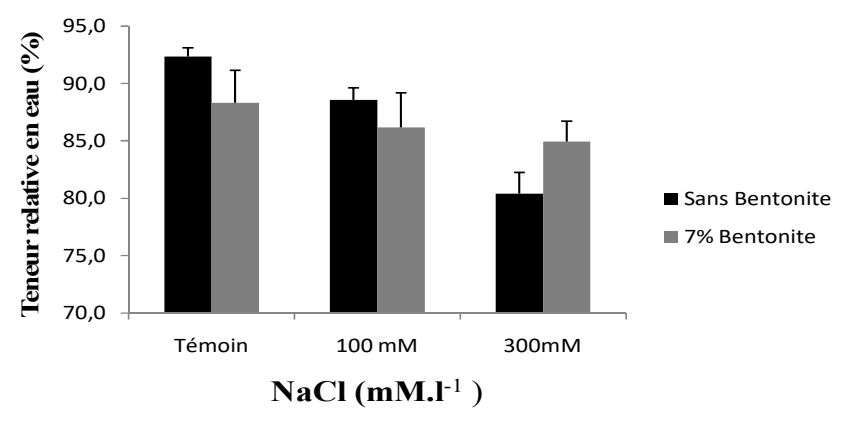

Fig 1. RWC des feuilles (\%) des plantes de Gombo (Abelmoschus esculentus L.) âgées de deux mois sous contrainte saline conduit sur substrat sans ou avec bentonite 
Table 2. Analyse de la variance des paramètres étudiés des plantes de gombo (Abelmoschus esculentus L.) âgées de deux mois sous contrainte saline conduit sur substrat bentonisé.

\begin{tabular}{lcccccc}
\hline Paramètres & \multicolumn{2}{c}{ Effet bentonite $(\mathrm{F} 1)$} & \multicolumn{2}{c}{ Effet salinité $(\mathrm{F} 2)$} & \multicolumn{2}{c}{ Interaction $(\mathrm{F} 1 \times \mathrm{F} 2)$} \\
\cline { 2 - 7 } & Test $\mathrm{f}$ & Probabilité & Test $\mathrm{f}$ & probabilité & Test $\mathrm{f}$ & probabilité \\
\hline RWC & 0.41 & 0.53 & 21.10 & 0.000 & 7.30 & 0.008 \\
\hline RWL30 min & 525.091 & 0.000 & 123.545 & 0.000 & 57.909 & 0.000 \\
\hline RWL60min & 429.081 & 0.000 & 38.297 & 0.000 & 54.243 & 0.000 \\
\hline DHS & 54.88 & 0.000 & 20.198 & 0.000 & 36.708 & 0.000 \\
\hline Proline & 32.997 & 0.000 & 93.924 & 0.000 & 18.709 & 0.000 \\
\hline Sucres soluble & 1.309 & 0.000 & 51.635 & 0.000 & 9.856 & 0.000 \\
\hline
\end{tabular}

\subsection{Déperdition de l'eau par transpiration (RWL)}

Les deux facteurs salinité et bentonite ont un effet significative sur la perte graduelle de l'eau $(\mathrm{p}<0.05)$ (Tableau 2), ainsi que, l'interaction des deux facteurs. A $30 \mathrm{mn}$, dans les conditions sans bentonite (Figure 2), une baisse remarquable de la transpiration des plantes stressées à 100 et $300 \mathrm{mM}$, dont les taux de réduction sont respectivement 17 et 53\% par rapport au témoin. A 60 minutes, cette diminution semble être moins rigoureuse avec des quantités d'eau perdues de $0,069 \mathrm{mg} / \mathrm{cm}^{2} / \mathrm{min}$ pour les témoins, 0.059 et $0.033 \mathrm{mg} / \mathrm{cm}^{2} / \mathrm{min}$ pour les plantes stressées à 100 et $300 \mathrm{mM}$ respectivement. En outre, sous le substrat bentonisé, la transpiration des plantes à 30 minutes subit une légère diminution à $100 \mathrm{mM} \mathrm{NaCl}$, au-delà les pertes en eau restent constantes (Figure 3). Après 60 minutes les taux de déperdition de l'eau sont significativement inférieures par rapport aux conditions du substrat sans bentonite. 


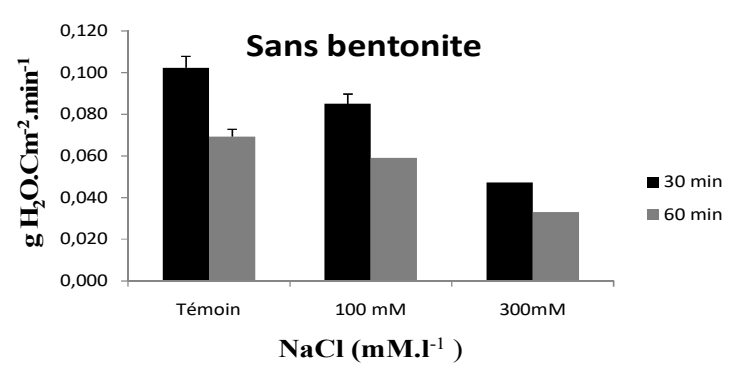

Fig 2. $\mathrm{RWL}\left(\mathrm{mg} \mathrm{H}_{2} \mathrm{O} \cdot \mathrm{Cm}^{-2} \cdot \mathrm{min}^{-1}\right)$ des feuilles des plantes de Gombo (Abelmoschus esculentus L.) âgées de deux mois cultivé sur substrat sans bentonite, soumises à différents traitements au $\mathrm{NaCl}$

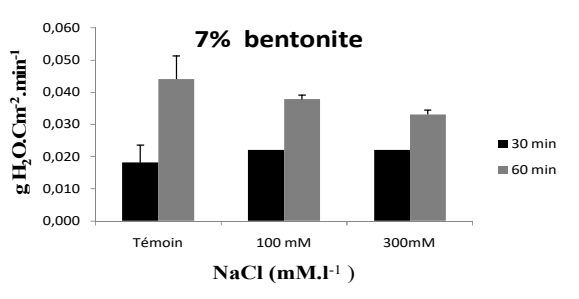

Fig 3. RWL (mg $\mathrm{H}_{2} \mathrm{O} \cdot \mathrm{Cm}^{-2} \cdot \mathrm{min}^{-1}$ ) des feuilles des plantes de Gombo (Abelmoschus esculentus L.) âgés de deux mois soumit à différents traitements de $\mathrm{NaCl}$ cultivées sur substrat bentonisé à 7\%

\subsection{Déficit hydrique de saturation (DHS)}

Les résultats illustrés dans le tableau 2, montre que le développement du DHS est en relation avec la salinité du milieu et le type de substrat $(\mathrm{p}<0.05, \mathrm{p}<0.05)$. L'interaction de ces deux facteur induit également un effet significatif sur le DHS $(\mathrm{p}<0.05)$. Nos résultats montrent que l'intensité du stress salin est à l'origine de l'augmentation des taux de DHS foliaire chez les plantes cultivées sans bentonite (Figure 4). Elles sont passées de 7,7 \% chez les témoins à plus de $17 \%$ chez les plantes stressées à $300 \mathrm{mM}$ de $\mathrm{NaCl}$ marquant une augmentation de $132 \%$. Par contre chez les plantes cultivées sous substrat bentonisé, le DSH des feuilles a subi une 
légère augmentation à $100 \mathrm{mM}$ de $\mathrm{NaCl}$, dont le taux d'augmentation est de $13 \%$.

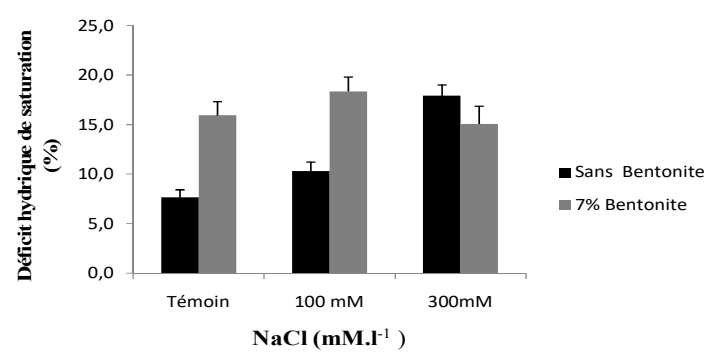

Fig 4. DHS des feuilles (\%) des plantes de Gombo (Abelmoschus esculentus L.) âgées de deux mois sous contrainte saline conduit sur substrat sans ou avec bentonite

\subsection{Teneurs en proline}

Les resultats indiquent qu'un effet salinité-substrat est hautement significatif sur l'accumulation de la proline; l'interaction de ces deux facteurs est également à l'origine des variations de ce paramètre (Tableau 2).

D'après la figure 5, la teneur en proline des feuilles augmente d'environ 63\% sous $100 \mathrm{mM}$ et de $81 \%$ sous $300 \mathrm{mM} \mathrm{NaCl}$. Néanmoins, dans les raciness, la teneur en proline a tendance à diminuer avec l'augmentation de la salinité dans le milieu pour atteindre $3.3 \mathrm{mg} . \mathrm{g}^{-1} \mathrm{PS}$ à100 $\mathrm{mM}$ et $2.48 \mathrm{mg} \cdot \mathrm{g}^{-1} \mathrm{PS}$ à $300 \mathrm{mM}$. Dans le milieu bentonisé (Figure 6), la teneur en proline aussi bien dans les feuilles que dans les racines a subi une augmentation significative seulement à $300 \mathrm{mM}$. Dans ce cas, on note des taux d'augmentation de $28 \%$ chez les feuilles et de $37 \%$ chez les racines par rapport aux témoins. 


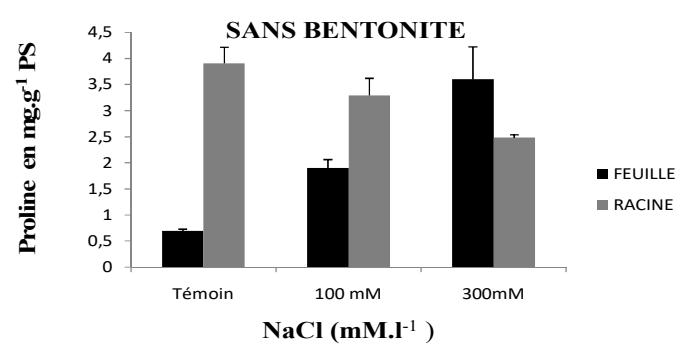

Fig 5. Variation de la proline (mg. $\left.\mathrm{g}^{-1} \mathrm{PS}\right)$ dans les feuilles et les racines des plantes de Gombo (Abelmoschus esculentus L.) âgées de deux mois sous contrainte saline et cultivées sur substrat sans bentonite

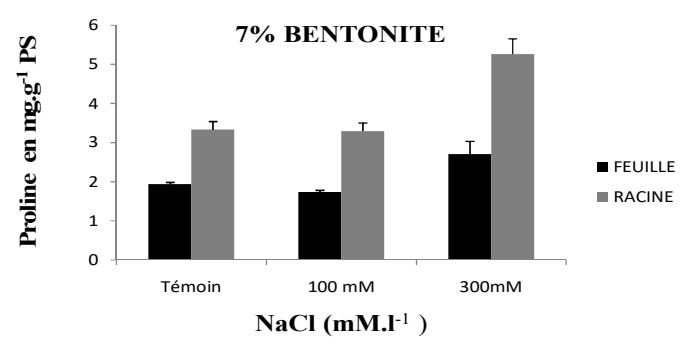

Fig 6. Variation de la proline $\left(\mathrm{mg} \cdot \mathrm{g}^{-1} \mathrm{PS}\right)$ dans les feuilles et les racines des plantes de Gombo (Abelmoschus esculentus L.) âgées de deux mois sous contrainte saline et cultivées sur substrat bentonisé à 7\%

L'analyse du tableau 3 révèle que l'accumulation de la proline dans les racines est plus importante que dans les feuilles chez les plantes témoins sans bentonite (SB) et celles traitées à 100 mM SB. A 300 mM SB la tendance de l'accumulation de cet acide aminé s'inverse dans le sens feuille racine. Pour atteindre un rapport $(\mathrm{F} / \mathrm{R})$ de 1,5. Tandis que, chez les plantes cultivées sous bentonite, les quantités de proline sont plus considérables en comparant avec le premier substrat, avec une accumulation qui se fait dans le sens racine-feuille. 
Table 3. Ratio des teneurs en proline des feuilles et des racines $(F / R)$ et les teneurs totales en proline (mg.g ${ }^{-1}$ PS) des plantes de Gombo (Abelmoschus esculentus L.) âgées de deux mois sous contrainte saline et cultivées sur substrat bentonisé à $7 \%$.

\begin{tabular}{|c|c|c|c|c|}
\hline & \multirow[b]{2}{*}{ Substrat } & \multirow[b]{2}{*}{ Témoin } & \multirow[b]{2}{*}{$100 \mathrm{Mm}$} & \multirow[b]{2}{*}{$300 \mathrm{mM}$} \\
\hline & & & & \\
\hline \multirow[t]{2}{*}{ Ratio (feuille/racine) } & Substrat sans bentonite (SB) & 0,18 & 0,58 & 1,45 \\
\hline & Substrat bentonisé à 7\% (B) & 0,58 & 0,52 & 0,51 \\
\hline \multirow{2}{*}{ 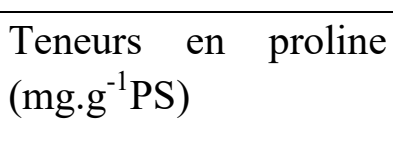 } & Substrat sans bentonite (SB) & 4.61 & 5.20 & 6.09 \\
\hline & Substrat bentonisé à 7\% (B) & 5.27 & 5.03 & 7.97 \\
\hline
\end{tabular}

\subsection{Teneurs en sucres soluble}

La salinité et la présence de bentonite ainsi que l'interaction entre les deux facteurs influent de manière hautement significative sur l'accumulation des sucres des feuilles et des racines $(\mathrm{p}<0.05, \mathrm{p}<0.05, \mathrm{p}<0.05)$ (Tableau 2). Hormis la concentration extrême de $\mathrm{NaCl}, 300 \mathrm{mM}$, avec un taux d'augmentation de $33 \%$, la salinité n'a pas affecté significativement l'accumulation des sucres solubles dans les feuilles des plantes dans le premier substrat (Figure 7), en comparant avec le témoin. Cette augmentation est moins importante chez les racines, où les teneurs en sucres sont passées de $0.14 \mathrm{mg} \cdot \mathrm{g}^{-1} \mathrm{PF}$ chez les témoins à $0.16 \mathrm{mg} \cdot \mathrm{g}^{-1}$ PF chez les plantes stressées à $300 \mathrm{mM}$. En présence de bentonite, la teneur en sucres solubles la plus élevée $0.33 \mathrm{mg} . \mathrm{g}^{-1} \mathrm{PF}$ est enregistrée chez les feuilles des plantes exposées à $300 \mathrm{mM}$ de $\mathrm{NaCl}$ (Figure 8). En revanche, la plus faible teneur en sucres (0.19 mg.g $\left.{ }^{-1} \mathrm{PF}\right)$ est enregistrée chez les racines des plantes témoins. 


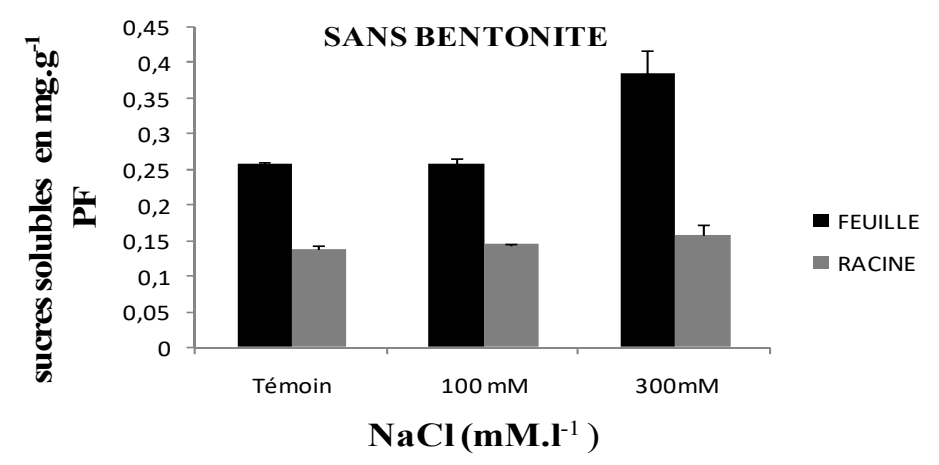

Fig 7. Teneurs en sucres solubles $\left(\mathrm{mg} \mathrm{g}^{-1} \mathrm{PF}\right)$ des feuilles et des racines des plantes de Gombo (Abelmoschus esculentus L.) âgées de deux mois sous contrainte saline et cultivées sur substrat sans bentonite

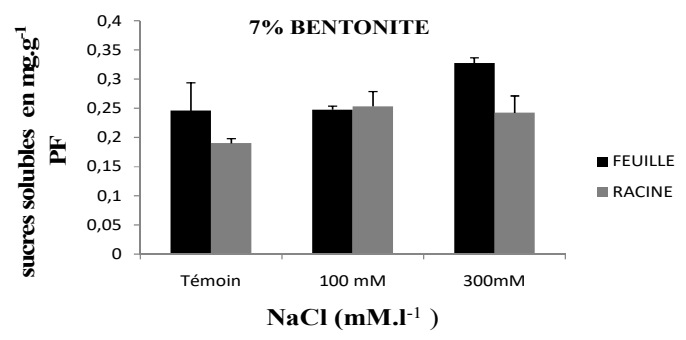

Fig 8. Teneurs en sucres solubles (mg. $\mathrm{g}^{-1} \mathrm{PF}$ ) des feuilles et des racines chez les plantes de Gombo (Abelmoschus esculentus L.) âgées de deux mois sous contrainte saline et cultivées sur substrat bentonisé à $7 \%$

D'après le tableau 4, dans le milieu bentonisé, les teneurs cumulées en sucres solubles des feuilles et des racines sont plus élevées $\left(0.43,0.56\right.$ et $\left.0.50 \mathrm{mg} . \mathrm{g}^{-1} \mathrm{PF}\right)$ comparativement à l'effet susbstrat sans bentonite $\left(0.39,0.39\right.$ et $\left.0.55 \mathrm{mg} \cdot \mathrm{g}^{-1} \mathrm{PF}\right)$. 
Table 4. Variation des teneurs en sucres solubles (mg. $\left.\mathrm{g}^{-1} \mathrm{PS}\right)$ des feuilles et des racines chez les plantes de Gombo (Abelmoschus esculentus L.) âgées de deux mois sous contrainte saline cultivées sur substrat bentonisé à 7\%

\begin{tabular}{lllll}
\cline { 2 - 5 } & \multicolumn{1}{c}{ Substrat } & Témoin & $100 \mathrm{mM}$ & $300 \mathrm{mM}$ \\
\hline Teneurs en sucres solubles & Substrat sans bentonite (SN) & 0.39 & 0.39 & 0.55 \\
\cline { 3 - 5 }$\left(\mathrm{mg} . \mathrm{g}^{-1} \mathrm{PS}\right)($ feuilles +racines) & Substrat bentonisé à 7\% (B) & 0.43 & 0.50 & 0.56 \\
\hline
\end{tabular}

\section{DISCUSSION}

Le stress salin modifie l'état hydrique de la plante [28], abaisse le contenu relatif en eau (RWC) des feuilles [10,11], limite les pertes en eau par transpiration des feuilles (RWL) et affaiblit l'assimilation hydrique par le système racinaire [12]. Le RWC des feuilles est un critère utilisé souvent pour apprécier l'état hydrique des plantes [29] et sa stabilité osmotique [30]. Les plantes exposées au stress salin augmentent leur résistance stomatique, afin de limiter la transpiration. Nos résultats indiquent une diminution du statut hydrique des plantes, observée principalement sur substrat sans bentonite chez les plantes stressées à 100 et 300 $\mathrm{mM}$ de $\mathrm{NaCl}$. Le comportement hydrique des plantes vis-à-vis de salinité est significatifement différent sous le substrat bentonisé à $7 \%$. La présence de bentonite dans les sols sableux améliore la capacité d'échange cationique [31], son aptitude à retenir l'eau et son transfert [32].

Dans des conditions de salinité, les plantes synthétisent la proline [33], responsable de l'ajustement osmotique et la protection de la cellule [34]. [35] a constaté que l'osmorégulation due à l'accumulation de certains acides aminés libres, est l'une des stratégies prédominantes utilisées par les plantes pour tolérer le stress salin. Le sel induit la stimulation de l'activité de la pyrroline-5-carboxylase synthétase chez Morus alba [36], chez Arachis hypogaea L. [37] et Vigna aconitifolia [38]. La proline s'accumule en plus grandes quantités que d'autres acides aminés dans les plantes stressées par le sel [39]. On a affirmée que les plantes qui Synthétisant de grandes quantités de proline sont plus tolérantes aux conditions de stress [40,41]. Dans 
notre étude les plantes de Gombo ont accumulé des quantités importantes de proline, particulièrement pour les plantes cultivées sur substrat bentonisé.

Par ailleurs, l'accumulation des sucres solubles, osmorégulateurs [42], admis comme indicateur de tolérance à la salinité [43]. Les sucres ont un effet important dans le processus d'ajustement osmotique [30]. Dans cette etude, les plantes de gombo montrent une forte accumulation des sucres, avec des teneurs plus élevées dans les plantes conduites en milieu bentonisé. Cette accumulation est corrélée positivement avec les concentrations en $\mathrm{NaCl}$ apporté. [44] Indique que le riz présente un comportement similaire sous des conditions de stresse salin [45] rapporte que l'accumulation des sucres est à l'origine de l'adaptation à la salinité ou la sécheresse.

\section{CONCLUSION}

L'exposition des plantes de gombo (Abelmoscus esculentus) âgée de deux mois, au stress salin s'est traduite par une diminution de contenu hydrique des feuilles accompagné d'une limitation dans la perte en eau. La proline et les sucres solubles sont accumulés au niveau des deux parties de la plante pour mettre en place, l'ajustement osmotique. La présence de bentonite sodique, riche en argile apporte des changements au niveau du comportement hydrique et biochimique de la plante, ceci est justifié à travers les résultats statistiques obtenus, à travers l'analyse de l'interaction entre la salinité et la bentonite.

\section{REFERENCES}

[1] Stoetzel E, Campmas E, Michel P, Bougariane B, Ouchaou B, Amani F, El Hajraoui M;A et Nespoulet R. Context of modern human occupations in North Africa: Contribution of the Témara caves data. Quaternary International., 2014, 320 : 143-161.

[2] Diack M. Diop T. et Ndiaye R. Restoration of Degraded Lands Affected by Salinization Process Under Climate Change Conditions: Impacts on Food Security in the Senegal River Valley, In : R. Lal, B. R. Singh, Dismas .L. Mwaseba, D. Kraybill, D. O. Hansen et L. O. Eik (Eds.), Sustainable Intensification to Advance Food Security and Enhance Climate Resilience in Africa. Springer Cham Heidelberg New York Dordrecht London, 2015, pp. 275-288. 
[3] Benbrahim KF, Ismaili M, Benbrahim SF, Tribak A. Problèmes de dégradation de l'environnement par la désertification et la déforestation : impact du phénomène au Maroc. Sécheresse., 2004, 15(4) : 307-320.

[4] Eynard A, Lala R, Keith D.W. In Encyclopedia of Soil Science, (CRC Press) Chapter: 323 (2006) 1538.

[5] Manchanda G et Garg N. Acta Physiol. Plant., 2008,30 :595.

[6] Faghire M, Bargaz A, Farissi M, Palma F, Mandri B, Lluch C, Tejera García NA, Herrera-Cervera JA, Oufdou K, Ghoulam C. Effect of salinity on nodulation, nitrogen fixation and growth of common bean (Phaseolus vulgaris L.) inoculated with rhizobial strains isolated from the haouz region of morocco. Symbiosis., 2011, 55: 69-75.

[7] Ben Naceur M, Rahmoune C, Sdiri H, Meddahi M.L et Selmi M. Effet du stress salin sur la germination, la croissance et la production en grains de quelques variétés maghrébines de blé. Science et Changements Planétaires /Sécheresse., 2001,12(3): 167-174.

[8] Mahajan S et Tuteja N. Cold salinity and drought stresses. An overview Archives of Biochemistry and Biophysics., 2005, 444 ( 2) :139-158.

[9] Turan MA, Elkarim AHA, Taban A et Taban S. Effect of salt stress on growth and ion distribution and accumulation in shoot and root of maize plant. Afr. J. Agric. Res., 2005, 5(7): 584-588.

[10] Kaya C, Hıggs D, Kırnak H et Taş I. Amelioratrive effect of calcium nitrate on cucumber and melon plants drip irrigated with saline water. Journal of Plant Nutrition., 2003, 26: 665-1681.

[11] Katerj1, N, Van Hoorn J.W, Hamdy A et Mastror1l11 M. Salinity effect on crop development and yield analysis of salt tolerance according to several classification methods. Agric. Water Management., 2003, 62: 37-66.

[12] Ngasamy P. World salinization with emphasis on Australia. Journal of Experimental Botany., 2006, $57:$ 1017-1023.

[13] Ashraf M. Biotechnological approach of improving plant salt tolerance using antioxidants as markers. Biotechnol Adv., 2009, 27:84-93. 
[14] Boriboonkaset T, Theerawitaya C, Yamada N, Pichakum A, Supaibulwatana K, Cha-um S, Takabe $\mathrm{T}$ et Kirdmanee C. Regulation of som metabolism-related genes, starch and soluble sugar contents, photosynthetic activities and yield attributes of two contrasting rice genotypes subjected to salt stress. Protoplasma., 2013, 250 (5) : 1157-1167.

[15] Slama I, Ben Rejeb K, Rouached A, Jdey A, Rabhi M, Talbi O, Debez A, Savouré A et Abdelly C. Presence of proline in salinized nutrient solution re-enforces the role of this amino acid in osmoregulation and protects lipid membrane peroxidation in Arabidopsis thaliana. Australian Journal of Crop Science., 2014, 8 (10) : 1367-1372.

[16] Berka S. et Aïd F. Réponses physiologiques des plants d'Argania spinosa (L.) Skeels soumis à un déficit hydrique édaphique. Sécheresse., 2009, 20(3) : 296-302.

[17] Saleem A, Ashraf $M$ et Akram NA. Salt $(\mathrm{NaCl})$ - induced modulation in some key physio-biochemical attributes in okra (Abelmoschus esculentus L.). J Agron Crop Sci., 2011, 197:202-13.

[18] Ashraf M, Akram NA, Arteca RN et Foolad MR. The physiological, biochemical and molecular roles of brassinosteroids and salicylic acid in plant processes and salt tolerance. Crit RevPlant Sci., 2010, 29:162-90.

[19] Akram NA, Ashraf M et Al-Qurainy F. Aminolevulinic acid-induced regulation in some key physiological attributes and activities of antioxidant enzymes in sunflower (Helianthus annuus L.) under saline regimes. Sci Hort., 2012, 142:143-8.

[20] Gaucher F. et S. Burdin, Géologie, géomorphologie et hydrologie des terrains sales. 1974.,- P.U.F., 234 p.

[21] El-Hady OA et El-Sherif AF. Egyptian Bentonitic deposits as soil amendements. Evaluation as conditioners for sandy soils. Egypt J Soil Sci., 1988, 28 : 205-14.

[22] Ashraf, M, Arfan, M, Ahmad, A. Salt tolerance in okra: ion relations and gas exchange characteristics. J. Plant Nutr., 2003, 26: 63-79.

[23] Clarke JM, Mc Caig TN. Evaluation of techniques for screening for drought resistance in wheat. Crop Science., 1982, 22 : 503-6.

[24] Heller R, Esnault R et Lance C.1998. L'eau dans la plante. In : Physiologie végétale DUNOD, ISBN 2100039911,315 p. 
[25] Bergman I et Loxley R. Two improved and simplified methods for the spectrophotometric determination of hydroxyproline. Anal. Chem., 1963, 35:1961.

[26] Shields R et Burnett W. Determination of protein-bound carbohydrate in serum by a nnodified anthrone method. Annals of Chemistry., 1960, 32: 885-886.

[27] Lewicki S. Evaluation de paramètres liés à l'état hydrique chez le blé dur (Triticum durum Desf) et l'orge (Hordeum vulgare L.) soumis à un déficit hydrique modéré, en vue d'une application à la sélection de génotypes tolérants. Thèse de doctorat, Ensa-Montpellier., 1993, $87 \mathrm{p}$.

[28] Fricke,W, Peters W. The biohysics of leaf growth in salt sressed barley. A study at the cell level. Plant Physiol ., 2002, $129: 388$.

[29] Meloni D.A, Gullota M.R, Martinez C.A et Oliva M.A. Les effet du stress salin sur la croissance, la reduction du Nitrate, de l'accumulation de la proline et de la glycine betaine chez Prosopis alba . Braz j. of plant Physiol ., 2004, $16: 1$.

[30] Morgan JM. Osmoregulation and waterin higher plants. Annu Rev Plant Physiol., 1984, 35:299-319.

[31] Dejou J. The specific surface of clay, its measurement, relationship with the CEC and its agronomic importance, in: Proceeding Symposium AFES, 1987, pp. 72-83.

[32] Raimund S et Dietmar S. Properties of soils under different types of management developed in a sandy substrate covering boulder clay at Mecklenburg (north eastern Germany), in: Sciences of Soils, 1996.

[33] Belkhodja M., 1996, Acta Bot. Gallica, 143(1), 21-28, doi:10.1080/12538078.19.10515315

[34] Nana R, Tamini Z et Sawadogo M. Effets d'un stress hydrique intervenu pendant le stade végétatif et la phase de floraison chez le gombo. Int. J. Biol. Chem. Sci., 2009, 3(5) : 1161-1170.

[35] Martino C.di, Delfine S, Pizzuto R, Loreto F \& Fuggi A. Free amino acids and glycine betaine in leaf osmoregulation of spinach responding to increasing salt stress. New Phytol., 2003, 158: 455-463. 
[36] Sudhakar C, Lakshmi A et Giridarakumar S. Changes in the antioxidant enzyme efficacy in two high yielding genotypes of mulberry (Morus alba L.) under $\mathrm{NaCl}$ salinity. Plant Sci., $2001,161: 613-619$.

[37] Girija C, Smith B.N et Swamy P.M. Interactive effects of sodium chloride and calcium chloride on the accumulation of proline and glycinebetaine in peanut (Arachis hypogaea L.). Env. Exp. Bot., 2002, 47 : 1-10.

[38] Hu C.A.A, Delauney A.J etVerma D.P.S. A bifunctional enzyme (1-pyrroline-5-carboxylase synthetase) catalyzes the first two steps in proline biosynthesis in plants. Proc. Natl. Acad. Sci. USA., 1992, 89 : 9354-9358.

[39] Abraham E, Rigo G, Szekely G, Nagy R, Koncz C \& Szabados L. Light-dependent induction of proline biosynthesis by abscisic acid and salt stress is inhibited by brassinosteroid in Arabidopsis. Plant Mol. Biol., 2003, 51: 363-372.

[40] Demiral Tet Türkan İ. Comparative lipid peroxidation, antioxidant defense systems and proline content in roots of two rice cultivars differing in salt tolerance. Environ. Exp. Bot., 2005, 53: 247-257.

[41] Mehdi H, Chaabouni A.C, Boujnah D et Boukhris M. 2010. The response of young pistachio trees grown under saline conditions depends on the rootstock, A no. XX, 2010-XIV GREMPA, Meeting on pistachios and almonds.

[42] Bouatrous Y. Water stress correlated with senescence in durum wheat (Triticum durum Desf). Advances in Environmental Biology., 2013, 7 (7) : 1306-1314.

[43] Munns R, James R. A et Lauchli A. Approaches to increasing the salt tolerance of wheat and other cereals. Journal of Experimental Botany., 2006, 57(5) :1025-1043.

[44] Dubey R.S et Singh A.K. Salinity induces accumulation of soluble sugars and alters the activity of sugar metabolising enzymes in rice plants. Biol. Plant., 1999, 42 : 233-239.

[45] Murakeozy E. P, Nagy Z, Duhaze C, Bouchereau A \& Tuba Z. Seasonal changes in the levels of compatible osmolytes in three halophytic species of inland saline vegetation in Hungary. J. Plant Physiol., 2003, $160: 395-401$.

[46] Hoagland D.R et Arnon D.I., 1938. The water-culture method for growing plants without soil. Berkeley, USA: College of Agriculture, University of California. 
[47] Entreprise Nationale des produits Miniers Non-Ferreux et des substances utiles, 1988.

\section{How to cite this article:}

Rahim Guealia H, Belkhodja M, Reguieg Yssaad HA, Babou FZ. Water and physiological responses of okra abelmoschus esculentus under saline stress grown on a bentonized substrate. J. Fundam. Appl. Sci., 2017, 9(3), 1395-1412. 